\title{
Plasticity-induced origami for assembly of three dimensional metallic structures guided by compressive buckling
}

Yan Shi ${ }^{1, \dagger}$, Fan Zhang ${ }^{1, \dagger}$, Kewang $\mathrm{Nan}^{2}$, Xueju Wang ${ }^{3}$, Juntong Wang ${ }^{2}$, Yijie Zhang ${ }^{2}$, Yutong Zhang $^{2}$, Haiwen Luan ${ }^{4}$, Keh-Chih Hwang ${ }^{1}$, Yonggang Huang ${ }^{4}$, John A. Rogers ${ }^{5, *}$, and Yihui Zhang ${ }^{1, *}$

${ }^{1}$ Department of Engineering Mechanics; Center for Mechanics and Materials; Applied Mechanics Laboratory; Tsinghua University, Beijing 100084, P.R. China.

${ }^{2}$ Department of Mechanical Science and Engineering, University of Illinois at Urbana-Champaign, Urbana, Illinois 61801, USA.

${ }^{3}$ Department of Materials Science and Engineering, Northwestern University, Evanston, IL 60208, USA.

${ }^{4}$ Departments of Civil and Environmental Engineering, Mechanical Engineering, and Materials Science and Engineering, Northwestern University, Evanston, IL 60208, USA

${ }^{5}$ Departments of Materials Science and Engineering, Biomedical Engineering, Chemistry, Mechanical Engineering, Electrical Engineering and Computer Science, and Neurological Surgery; Center for Bio-Integrated Electronics; Simpson Querrey Institute for Nano/biotechnology; Northwestern University, Evanston, IL 60208, USA

$\dagger$ These authors contributed equally to this work.

Correspondence and requests for materials should be addressed to John A. Rogers (email: jrogers@illinois.edu) or Yihui Zhang (email: yihuizhang@tsinghua.edu.cn). 


\section{ABSTRACT}

Development of origami-inspired routes to assembly of three dimensional structures is an area of growing activity in scientific and engineering research communities due to fundamental interest in mathematical topics in topology and to the potential for practical applications in areas ranging from advanced surgical tools to systems for space exploration. Recently reported approaches that exploit the controlled, compressive buckling of 2D precursors induced by dimensional change in an underlying elastomer support offer broad versatility in material selection (from polymers to device grade semiconductors), feature sizes (from centimeters to nanometers), topological forms (open frameworks to closed form polyhedra) and shape controllability (dynamic tuning of shape), thereby establishing a promising avenue to autonomic assembly of complex 3D systems. Localization of origami-like folding deformations at targeted regions can be achieved through the use of engineered, spatial variations in the thicknesses of the 2D precursors. While this approach offers high levels of control in the targeted formation of creases, creating the necessary thickness variations requires a set of additional processing steps in the fabrication. This paper presents an alternative, and complementary, approach that exploits controlled plastic deformation in the precursors, as validated in a comprehensive set of experimental and theoretical studies. Specifically, plasticity and strain localization can be used to dramatically reduce the bending stiffness at targeted regions, to form well-defined creases as mountain or valley folds during the $2 \mathrm{D}$ to $3 \mathrm{D}$ geometrical transformation process. The content begins with studies of a model system that consists of a $2 \mathrm{D}$ precursor in the form of a straight ribbon with reduced widths at certain sections. The results illustrate the important 
role of plasticity in the course of folding, in such a manner that dictates the final 3D layouts. A broad range of complex 3D shapes, achieved in both the millimeter-scale and the mesoscale structures (i.e. micron to sub-millimeter), demonstrate the power of these ideas.

Keywords: Buckling; Origami assembly; 3D metallic structures; Plasticity. 


\section{Introduction}

Origami, the ancient Japanese art of paper folding, has recently attracted significant interest in various research communities, due to its combined content in areas ranging from mathematical concepts in topology to engineering strategies in device design. The latter encompasses broad ranging types of functionality and dimensional scale, from deformable batteries, ${ }^{[1,2]}$ biomedical devices, ${ }^{[3-7]}$ stretchable electrodes, ${ }^{[8-10]}$ microelectromechanical (MEMS) and nanoelectromechanical (NEMS) systems, ${ }^{[11-14]}$ to metamaterials ${ }^{[15-18]}$ and DNA folding structures. ${ }^{[19-21]}$ Development of inverse-problem algorithms that can yield the crease distributions and folding sequences to achieve desired 3D structure represents an essential goal in this area. ${ }^{[22-24]}$ In one example, researchers utilize the concept of lattice kirigami, ${ }^{[23,25-27]}$ as a variant of origami that involves both cutting and folding, to realize the complex 3D structures. Another key issue is in the identification of schemes to achieve automated folding actuation and improved control over the geometries 3D structures. Programmable shape changes can be realized using self-actuating materials, i.e., shape memory alloys/polymers, ${ }^{[28-33]}$ hydrogels,${ }^{[34-37]}$ and liquid crystal elastomers. ${ }^{[38]}$ These schemes are not, however, directly applicable to the classes of highly developed materials and micro/nanofabrication methods found in state-of-the-art microsystems technologies, such as those in integrated circuits, photonic devices, optoelectronic systems, sensors, microelectromechanical components and many others. Approaches that rely on residual stresses in thin films ${ }^{[4,39-41]}$ and/or capillary forces ${ }^{[42-45]}$ can enable origami-type assembly in some of these cases, but the range of $3 \mathrm{D}$ topologies that can be achieved is limited, precise folding angles can be difficult to control and the structures cannot typically be adjusted after fabrication. ${ }^{[4,39,40,42,44,45]}$ Recently, Yan et al. ${ }^{[46,47]}$ introduced a mechanically-guided 
approach to automated origami assembly of 3D mesostructures (with critical dimensions from nanometers to centimeters) in broad classes materials, including the types of high-performance thin films that are widely adopted in the semiconductor industry. This assembly scheme allows deterministic control of the 3D configurations, in a continuous and reversible manner, including over the intermediate states that occur throughout the $2 \mathrm{D}$ to $3 \mathrm{D}$ transformation. A well-designed spatial variation of thickness in the initial 2D structures represents the key control variable that allows localization of folding creases during a compressive buckling process that is induced in a $2 \mathrm{D}$ precursor by relaxing prestrain in an underlying elastomer substrate. The required non-uniform distribution of thickness, however, complicates the fabrication process and reduces the yield, leading to certain constraints in practical applications. Here, we introduce a set of concepts that exploit plasticity and strain localization achieved through optimization of the lateral geometries of 2D precursors with uniform thicknesses, to enable origami assembly in materials selected for their plastic yielding properties. Demonstrations of this concept include around twenty 3D structures made of copper thin films, with the thickness from a few microns to several tens of microns, formed through either unidirectional or bidirectional folding.

\section{Results and Discussions}

Figure 1 illustrates the design concepts in a representative straight ribbon structure with uniform thickness. The square anchors (i.e., bonding region, length $L_{b}$; red, Fig. 1(a)) strongly bond to a uniaxially pre-stretched elastomer substrate. Based on the mechanisms of

compressive buckling, ${ }^{[48,49]}$ the forces associated with the release of pre-stretch in the elastomer transform the metallic 2D precursors into 3D configurations. Here, the width of 
the ribbon narrows at selected regions, including two ends and one middle section, to decrease the local bending stiffness, thereby leading to strain localization. This localization, when controlled to lead to plastic yielding but not failure, decreases the local bending stiffness substantially due to material softening, thereby leading to the formation of folding creases during compression. The geometric parameters, including the width ratio $\left(w_{c} / w\right)$ and the dimensionless thickness $(t / L)$ normalized by the ribbon length, can be finely tuned to achieve a desired level of strain localization. A comparative study involving copper (yielding strain, $\sim 0.3 \%$; thickness, $30 \mu \mathrm{m}$ ) and polyethylene terephthalate (PET, yielding strain, > 10\%; thickness, $42 \mu \mathrm{m}$ ) films highlights the effects of plasticity. The $2 \mathrm{D}$ precursors in PET and copper have the same shapes, and the in-plane dimensions have the same ratio (1.4) as that of the thicknesses. Figure 1(b) shows results of experiment and FEA (See more details of experiment and FEA in Supplementary Information) on the buckled structures of the ribbons under different levels of prestrain. Folding clearly occurs at the desired crease locations in the copper structures, due to strain localization as indicated by the yielding region (red in FEA). By contrast, the PET ribbons exhibit relatively smooth bending deformations, since the materials undergo elastic deformation throughout the compression. FEA results agree with the experiments in all cases.

The extent of folding deformations can be characterized by the ratio of minimum curvature radius $\left(\rho_{c}\right)$ in the ribbons to the crease length $\left[L_{c}\right.$ in Fig. 1(a)]. To set a quantitative guideline for the design, a threshold of $\left(\rho_{c} / L_{c}\right)$ can be defined to characterize the initiation of evident folding deformations. The minimum prestrain $\left(\varepsilon_{c}\right)$ necessary to induce strong folding can be then determined for a given threshold [e.g., 0.5 in Fig. 1(c)]. FEA 
calculations show that the minimum prestrain decreases with increasing the film thickness, mainly due to the intensified strain localization at the creases. As the film thickness exceeds a certain value $\left[\sim 20 \mu \mathrm{m}\right.$ in Fig. 1(c)], the minimum prestrain $\left(\varepsilon_{c}\right)$ approaches a constant value $(\sim 9 \%)$. The calculations also indicate that the PET ribbons do not satisfy the above criterion for the scenarios shown in Fig 1(b).

In origami assembly, the folding angle $(\theta)$ [in Fig. 1(b)] represents a key parameter. When folding deformations dominate at the creases, it is reasonable to assume that the wider parts (width $w$ ) remain straight and that deformations in the bonding locations are negligible. With these assumptions, the folding angle $(\theta)$ can be determined from geometric analyses, as a function of the prestrain $\left(\varepsilon_{p r e}\right)$ and the geometric parameters, i.e.,

$$
\theta=2 \arcsin \left[\left(1-L_{b} \varepsilon_{\text {pre }} / L\right) /\left(1+\varepsilon_{\text {pre }}\right)\right], \quad \varepsilon_{\text {pre }}<L / L_{b}
$$

where $L$ and $L_{b}$ are lengths of the ribbon and bonding anchor, respectively, as shown in Fig. 1(a). Figure 1(d) demonstrates that this solution [Eq. (1)] agrees well with FEA and experimental findings for the design in Fig. 1(b). These results indicate that an enhanced level of prestrain increases the extent of folding.

Without the plastic effect, an evident folding deformation is only possible by adopting a very small width ratio to achieve the desired reduction of bending stiffness in the design, as illustrated in Fig. 1(e). For the elastic PET ribbons transformed by prestrains of $32 \%$, the structures undergo relatively smooth bending deformations for width ratios from 0.2 to 0.5 , and do not satisfy the above criterion for the initiation of evident folding deformations. The flatness of the two segments in the PET structure becomes comparable to the copper structure in Fig. 1(b) (with a width ratio of 0.5 ), only when the width ratio is 0.1 or smaller. 
Such a small width ratio results in an extremely narrow connection between the adjacent components, leading to mechanical fragility and other challenges during the processing. This limitation can be avoided by exploiting plasticity in the metallic structures, as evidenced by the large width ratios [e.g., 0.5 in Fig. 1(a)-(d) and (f)].

Figure 1(f) shows buckling deformations of a metallic ribbon with an asymmetric distribution of creases, for prestrains ranging from $0 \%$ to $90 \%$. The optical images and FEA predictions exhibit good agreement. Here, two angles $\left(\theta_{1}\right.$ and $\left.\theta_{2}\right)$ can be introduced to define the configurations of the buckled ribbons. These angles can be tuned continuously by changing the prestrain levels, leading to the formation of different forms of triangles, e.g., right triangles for $\varepsilon_{p r e}=\sim 25 \%$ or $61 \%$; acute triangles for $25 \%<\varepsilon_{\text {pre }}<61 \%$; obtuse triangles for $0 \%<\varepsilon_{\text {pre }}<25 \%$ and $\varepsilon_{\text {pre }}>61 \%$.

Figure 2 highlights the use of this design concept in several metallic structures that involve bi-directional folding generated by equal biaxial prestrain in the elastomer substrate. As before, the effects of plasticity can be observed by comparing copper (30 $\mu \mathrm{m})$ and PET (42 $\mu \mathrm{m})$ films in experiment and modeling. Figure 2(a) shows a precursor design that consists of five squares, with a width ratio of 0.53 , leading to the formation of a cubic box. For the copper structure [middle top and right top frames in Fig. 2(a)], the bending stiffnesses of the crease regions are much smaller than those of other areas, and therefore, the squares adjacent to the creases undergo negligible bending deformations, as evidenced by the nearly flat configurations after full release of the prestrain (86\%). In contrast, the squares in the PET structure [middle bottom and right bottom frames in Fig. 2(a)] undergo continuous bending deformations, and localized folding deformations do not appear. For the design 
(width ratio, 0.57) in Fig. 2(b), full release of the prestrain (100\%) leads to the formation of an inverted pyramid. Again, the surfaces are flat and curved in the copper and PET structures, respectively. The differences are even more pronounced at the crease regions, as shown by the insets of the right frames. Concepts inspired by kirigami ${ }^{[50,51]}$ involve additional cuts in the $2 \mathrm{D}$ precursor, which can expand the accessible range of $3 \mathrm{D}$ structures. $^{[46]}$ Figure 2(c) shows an example of lattice kirigami ${ }^{[23,25]}$ consisting of hexagon and rectangular parts connected by creases with a width ratio of 0.45 . Upon release of $18 \%$ prestrain, a hexagonal array of hexagonal prisms forms in the copper structure. This set of comparisons also demonstrates more evident folding deformations in the copper structure than those in the PET structure. In all of these examples, the FEA predictions agree well with experiments.

This strategy affords great versatility in the design of 3D structures. Figure 3(a) and (b) shows two simple origami examples that consist of both ribbons and membranes. Using $72 \%$ biaxial prestrain, 3D "table" structures with straight or tilted legs can be formed, as shown in Fig. 3(a) and (b), respectively. The latter involves rotational deformations in the 3D structures during assembly, due to the anti-symmetric arrangement of the legs relative to the table center. Increasing the number of the ribbons leads to the formation of a Greek Doric style architecture [Fig. 3(c)]. Combination of design concepts inspired by kirigami and origami enables the formation of more complex 3D configurations, as shown in Fig. 3(d)-(j). Each of these structures is identified with a descriptive name, i.e., windmill [Fig. 3(d)], tent [Fig. 3(e)], altar [Fig. 3(f)], plane [Fig. 3(g)], asparagus pea [Fig. 3(h)], five-pointed star [Fig. 3(i)], and soccer-ball [Fig. 3(j)]. The 3D configurations predicted by 
FEA agree well with the experiments.

This strategy is also applicable to the construction of origami structures at the mesoscale. Figure 3(k)-(n) presents several examples of 3D mesostructures formed from 2D precursors made of copper films (thickness, $3 \mu \mathrm{m}$; see more details of experiment in Supplementary Information). The layouts of the 2D precursors are similar to the millimeter-scale examples except for relatively large anchor regions designed to avoid delamination. Corresponding FEA results show good agreement with the experiments. In all of the examples presented here, metal plasticity plays a notable role in maintaining the planar shape of the surfaces in the 3D structures. Quantitative analyses based on FEA show that the maximum strain always occurs at the crease regions, and is typically in the range of 5\% to $8 \%$, which is well below the fracture threshold $(\sim 10 \%)$ of copper.

\section{Conclusions}

In summary, this work introduces a set of ideas that exploit plasticity and strain localization to achieve origami assembly of 3D metallic structures with uniform thicknesses from corresponding 2D precursors. Combined experimental and theoretical studies illustrate the utility of these strategies and highlight explicitly the important role of plasticity. Demonstrations of this concept include a broad set of 3D structures made of copper thin films, with thicknesses from a few microns to several tens of microns, formed through either unidirectional or bidirectional folding. The resulting 3D metallic structures have many potential applications, e.g., in broad-band reflectors and devices for blocking electromagnetic wave. 


\section{Acknowledgements}

Y.Z. acknowledges the support from the National Natural Science Foundation of China (Grant No. 11672152) and the Thousand Young Talents Program of China. Y.H. acknowledges the support from the NSF (Grant Nos. DMR1121262, CMMI1300846, and CMMI1534120). Y.H. and J.A.R. acknowledge the support from the NSF (Grant No. CMMI1400169) and the NIH (Grant No. R01EB019337).

\section{References}

[1] Z. Song, T. Ma, R. Tang, Q. Cheng, X. Wang, D. Krishnaraju, R. Panat, C. K. Chan, H. Yu, H. Jiang, Nat. Commun. 5 (2014) 3140.

[2] Q. Cheng, Z. Song, T. Ma, B. B. Smith, R. Tang, H. Yu, H. Jiang, C. K. Chan, Nano Lett. 13 (2013) 4969.

[3] M. Jamal, S. S. Kadam, R. Xiao, F. Jivan, T.-M. Onn, R. Fernandes, T. D. Nguyen, D. H. Gracias, Adv. Healthc. Mater. 2 (2013) 1142.

[4] T. G. Leong, C. L. Randall, B. R. Benson, N. Bassik, G. M. Stern, D. H. Gracias, Proc. Natl. Acad. Sci. 106 (2009) 703.

[5] M. R. Yu, Y. Huang, J. Ballweg, H. Shin, M. H. Huang, D. E. Savage, M. G. Lagally, E. W. Dent, R. H. Blick, J. C. Williams, ACS Nano 5 (2011) 2447.

[6] A. A. Solovev, S. Sanchez, M. Pumera, Y. F. Mei, O. G. Schmidt, Adv. Funct. Mater. 20 (2010) 2430.

[7] C. L. Randall, E. Gultepe, D. H. Gracias, Trends Biotechnol. 30 (2012) 138.

[8] F. Xu, W. Lu, Y. Zhu, Acs Nano 5 (2011) 672.

[9] Y. L. Chen, Y. L. Liu, Y. Yan, Y. Zhu, X. Chen, J. Mech. Phys. Solids 95 (2016) 25.

[10] Y. Liu, Z. Yan, Q. Lin, X. L. Guo, M. D. Han, K. Nan, K. C. Hwang, Y. G. Huang, Y. H. Zhang, J. A. Rogers, Adv. Funct. Mater. 26 (2016) 2909.

[11] J. A. Rogers, Y. Huang, O. G. Schmidt, D. H. Gracias, MRS Bull. 41 (2016) 123.

[12] D. Bishop, F. Pardo, C. Bolle, R. Giles, V. Aksyuk, J. Low Temp. Phys. 169 (2012) 386.

[13] W. Piyawattanametha, P. R. Patterson, D. Hah, H. Toshiyoshi, M. C. Wu, J. Microelectromech. S. 14 (2005) 1329.

[14] S. Yao, Y. Zhu, Adv. Mater. 27 (2015) 1480.

[15] J. L. Silverberg, A. A. Evans, L. McLeod, R. C. Hayward, T. Hull, C. D. Santangelo, I. Cohen, Science 345 (2014) 647.

[16] E. T. Filipov, T. Tachi, G. H. Paulino, Proc. Natl. Acad. Sci. 112 (2015) 12321. 
[17] M. Eidini, G. H. Paulino, Sci. Adv. 1 (2015) e1500224.

[18] C. Cao, Y. Feng, J. Zang, G. P. López, X. Zhao, Extreme Mech. Lett. 4 (2015) 18.

[19] Y. Ke, S. M. Douglas, M. Liu, J. Sharma, A. Cheng, A. Leung, Y. Liu, W. M. Shih, H. Yan, J. Am. Chem. Soc. 131 (2009) 15903.

[20] B. Saccà, C. M. Niemeyer, Angew. Chem. Int. Edit. 51 (2012) 58.

[21] Y. He, T. Ye, M. Su, C. Zhang, A. E. Ribbe, W. Jiang, C. Mao, Nature 452 (2008) 198.

[22] L. H. Dudte, E. Vouga, T. Tachi, L. Mahadevan, Nat. Mater. 15 (2016) 583.

[23] D. M. Sussman, Y. Cho, T. Castle, X. Gong, E. Jung, S. Yang, R. D. Kamien, Proc. Natl. Acad. Sci. 112 (2015) 7449.

[24] F. Wang, H. Gong, X. Chen, C. Q. Chen, Sci. Rep. 6 (2016) 33312.

[25] T. Castle, Y. Cho, X. Gong, E. Jung, D. M. Sussman, S. Yang, R. D. Kamien, Phys. Rev. Lett. 113 (2014) 245502.

[26] T. Castle, D. M. Sussman, M. Tanis, R. D. Kamien, Sci. Adv. 2 (2016) e1601258.

[27] Z. M. Song, X. Wang, C. Lv, Y. H. An, M. B. Liang, T. Ma, D. He, Y. J. Zheng, S. Q. Huang, H. Y. Yu, H. Q. Jiang, Sci. Rep. 5 (2015) 10988.

[28] P. Krulevitch, A. P. Lee, P. B. Ramsey, J. C. Trevino, J. Hamilton, M. A. Northrup, J. Microelectromech. S. 5 (1996) 270.

[29] E. Hawkes, B. An, N. M. Benbernou, H. Tanaka, S. Kim, E. D. Demaine, D. Rus, R. J. Wood, Proc. Natl. Acad. Sci. 107 (2010) 12441.

[30] M. Behl, M. Y. Razzaq, A. Lendlein, Adv. Mater. 22 (2010) 3388.

[31] J. Leng, X. Lan, Y. Liu, S. Du, Prog. Mater Sci. 56 (2011) 1077.

[32] S. M. Felton, M. T. Tolley, B. Shin, C. D. Onal, E. D. Demaine, D. Rus, R. J. Wood, Soft Matter 9 (2013) 7688.

[33] Q. Zhang, J. Wommer, C. O'Rourke, J. Teitelman, Y. Tang, J. Robison, G. Lin, J. Yin, Extreme Mech. Lett. (2016) in press.

[34] S. T. Lin, H. Yuk, T. Zhang, G. A. Parada, H. Koo, C. J. Yu, X. H. Zhao, Adv. Mater. 28 (2016) 4497.

[35] M. Jamal, A. M. Zarafshar, D. H. Gracias, Nat. Commun. 2 (2011) 527.

[36] K. Malachowski, M. Jamal, Q. Jin, B. Polat, C. J. Morris, D. H. Gracias, Nano Lett. 14 (2014) 4164.

[37] T. S. Shim, S.-H. Kim, C.-J. Heo, H. C. Jeon, S.-M. Yang, Angew. Chem. Int. Edit. 51 (2012) 1420.

[38] T. H. Ware, M. E. McConney, J. J. Wie, V. P. Tondiglia, T. J. White, Science 347 (2015) 982.

[39] W. J. Arora, A. J. Nichol, H. I. Smith, G. Barbastathis, Appl. Phys. Lett. 88 (2006) 053108.

[40] J. S. Randhawa, T. G. Leong, N. Bassik, B. R. Benson, M. T. Jochmans, D. H. Gracias, J. Am. Chem. Soc. 130 (2008) 17238.

[41] N. Bassik, G. M. Stern, D. H. Gracias, Appl. Phys. Lett. 95 (2009) 091901.

[42] C. Py, P. Reverdy, L. Doppler, J. Bico, B. Roman, C. N. Baroud, Phys. Rev. Lett. 98 (2007) 156103.

[43] X. Guo, H. Li, B. Y. Ahn, E. B. Duoss, K. J. Hsia, J. A. Lewis, R. G. Nuzzo, Proc. Natl. Acad. Sci. 106 (2009) 20149.

[44] R. R. A. Syms, E. M. Yeatman, Electron. Lett. 29 (1993) 662. 
[45] S. Pandey, M. Ewing, A. Kunas, N. Nghi, D. H. Gracias, G. Menon, Proc. Natl. Acad. Sci. 108 (2011) 19885.

[46] Z. Yan, F. Zhang, J. Wang, F. Liu, X. Guo, K. Nan, Q. Lin, M. Gao, D. Xiao, Y. Shi, Y. Qiu, H. Luan, J. H. Kim, Y. Wang, H. Luo, M. Han, Y. Huang, Y. Zhang, J. A. Rogers, Adv. Funct. Mater. 26 (2016) 2629.

[47] Z. Yan, F. Zhang, F. Liu, M. Han, D. Ou, Y. Liu, Q. Lin, X. Guo, H. Fu, Z. Xie, M. Gao, Y. Huang, J. Kim, Y. Qiu, K. Nan, J. Kim, P. Gutruf, H. Luo, A. Zhao, K.-C. Hwang, Y. Huang, Y. Zhang, J. A. Rogers, Sci. Adv. 2 (2016) e1601014.

[48] S. Xu, Z. Yan, K.-I. Jang, W. Huang, H. Fu, J. Kim, Z. Wei, M. Flavin, J. McCracken, R. Wang, A. Badea, Y. Liu, D. Xiao, G. Zhou, J. Lee, H. U. Chung, H. Cheng, W. Ren, A. Banks, X. Li, U. Paik, R. G. Nuzzo, Y. Huang, Y. Zhang, J. A. Rogers, Science 347 (2015) 154.

[49] Y. Zhang, Z. Yan, K. Nan, D. Xiao, Y. Liu, H. Luan, H. Fu, X. Wang, Q. Yang, J. Wang, W. Ren, H. Si, F. Liu, L. Yang, H. Li, J. Wang, X. Guo, H. Luo, L. Wang, Y. Huang, J. A. Rogers, Proc. Natl. Acad. Sci. 112 (2015) 11757.

[50] Y. Cho, J. H. Shin, A. Costa, T. A. Kim, V. Kunin, J. Li, S. Y. Lee, S. Yang, H. N. Han, I. S. Choi, D. J. Srolovitz, Proc. Natl. Acad. Sci. 111 (2014) 17390.

[51] Y. C. Tang, G. J. Lin, L. Han, S. G. Qiu, S. Yang, J. Yin, Adv. Mater. 27 (2015) 7181. 


\section{Figure captions}

Figure 1. Conceptual illustration and demonstrative examples of $3 \mathrm{D}$ origami structures assembled from 2D precursors by compressive buckling. (a) Top view of symmetric (left) and asymmetric (right) 2D precursors, in which the red color represents the bonding regions. (b) Optical images and corresponding FEA results for straight ribbon structures (thickness, 30 $\mu \mathrm{m}$ for copper and $42 \mu \mathrm{m}$ for PET) under six different levels of prestrain. (c) Critical prestrain $\left(\varepsilon_{c}\right)$ to initiate evident folding deformation for a symmetric ribbon design with a wide range of thicknesses $(t)$. (d) Folding angle $(\theta)$ versus the prestrain $\left(\varepsilon_{p r e}\right)$ for the symmetric ribbon design with a fix thickness $(30 \mu \mathrm{m})$. (e) Optical images and corresponding FEA results for symmetric PET ribbons $(42 \mu \mathrm{m})$ with a range of width ratios under a fixed prestrain (32\%). (f) Optical images and corresponding FEA results for copper ribbons with an asymmetric design that are under six different levels of prestrain. $\theta_{1}$ and $\theta_{2}$ denote the folding angle of the shorter ribbon segment and the angle between the two segments of the ribbon. In Fig. 1(b) and (e), the color in the FEA results corresponds to the magnitude of maximum principal strain $\left(\varepsilon_{\text {max-principal }}\right)$. Scale bars, $4 \mathrm{~mm}$.

Figure 2. Representative examples that illustrate the effect of plasticity on the assembly process. (a) 2D precursor (left) and corresponding 3D structures (middle, experiment results; right, FEA results) that resemble a cubic box. Both copper and PET films were used to construct the 3D structures, highlighting the plastic effect. (b) and (c) Similar results that correspond to the configurations of an inverted pyramid and a hexagonal array of hexagon prisms. Scale bars, $4 \mathrm{~mm}$. 
Figure 3. Experimental and computational studies of origami structures formed at both the millimeter-scale and mesoscale. 2D precursors, optical images and FEA simulations for fourteen structures formed with the use of equal biaxial pre-stretch in the elastomer substrate. Structures in (a)-(j) are made of thick copper films $(30 \mu \mathrm{m})$, and those in (k)-(n) are made of thin copper films (3 $\mu \mathrm{m})$. Scale bars, $4 \mathrm{~mm}$ in (a)-(j) and $400 \mu \mathrm{m}$ in (k)-(n), respectively. 

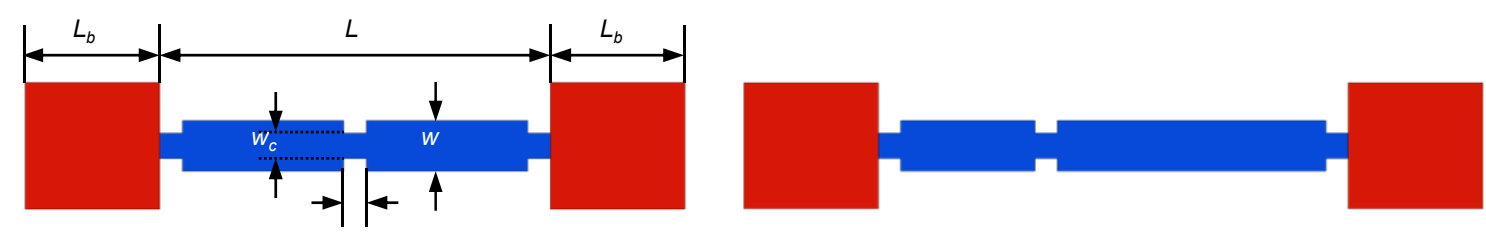

b

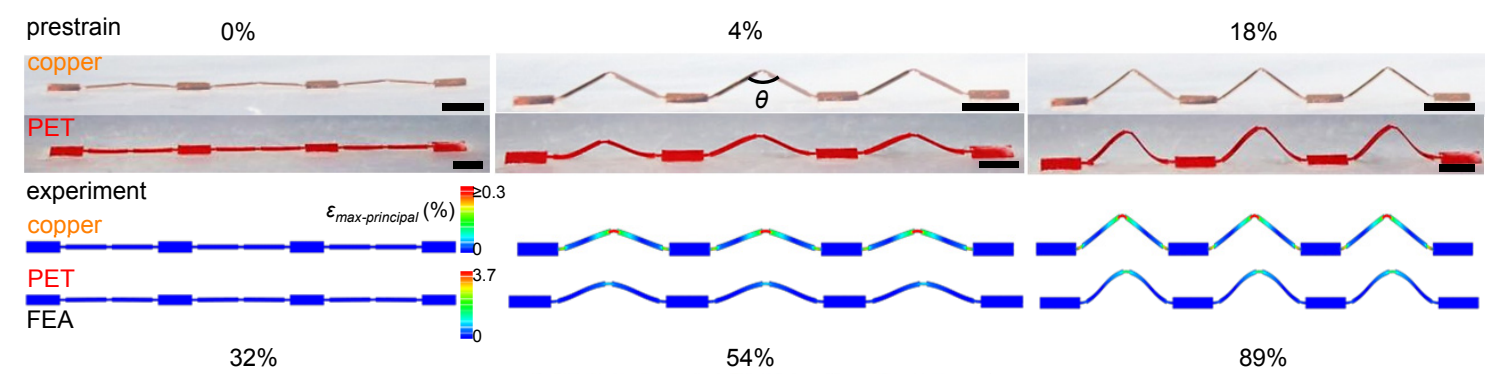

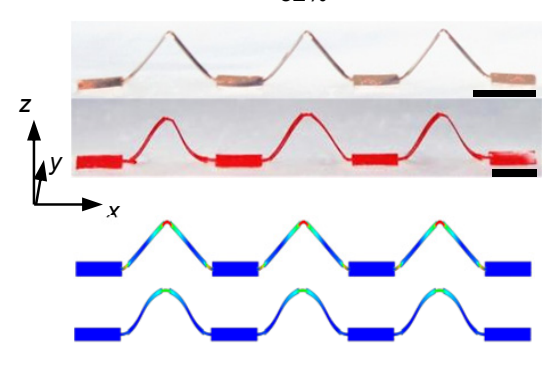

c

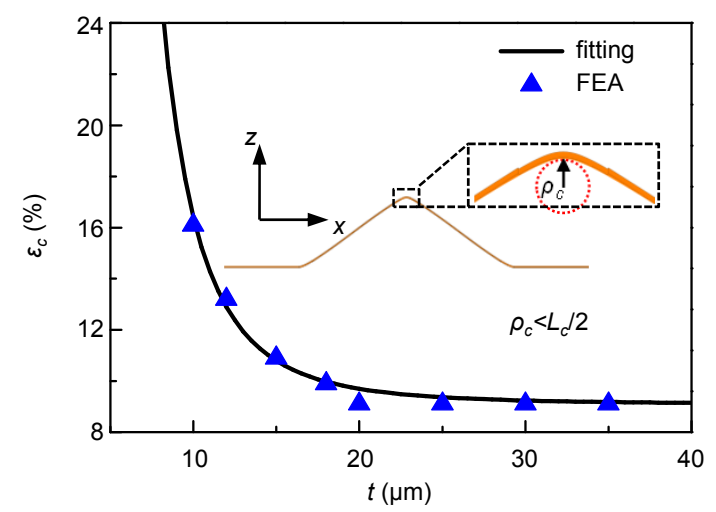

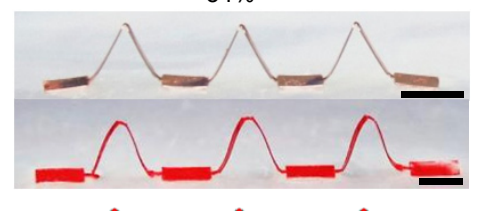

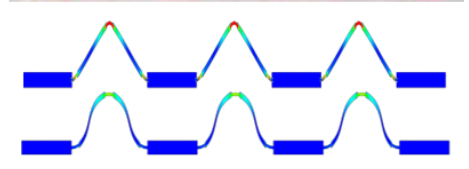

d

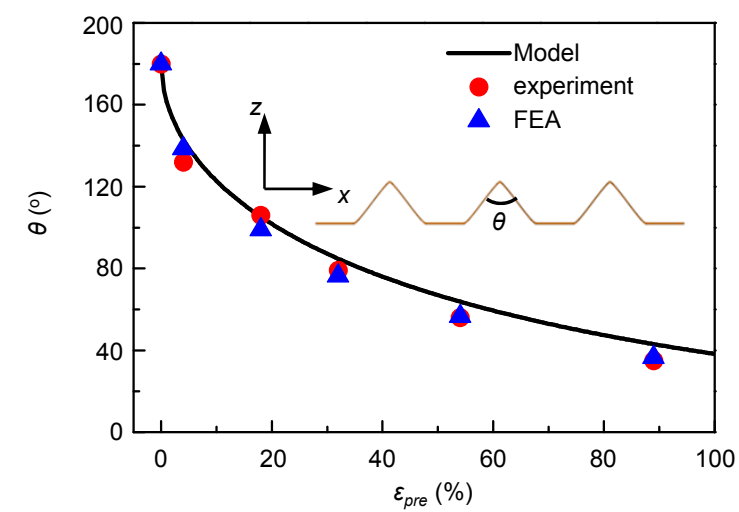

e width ratio 0.5

$\underset{x}{y \text { experiment }} \varepsilon_{\text {max-orincipal }}(\%)=3.4$
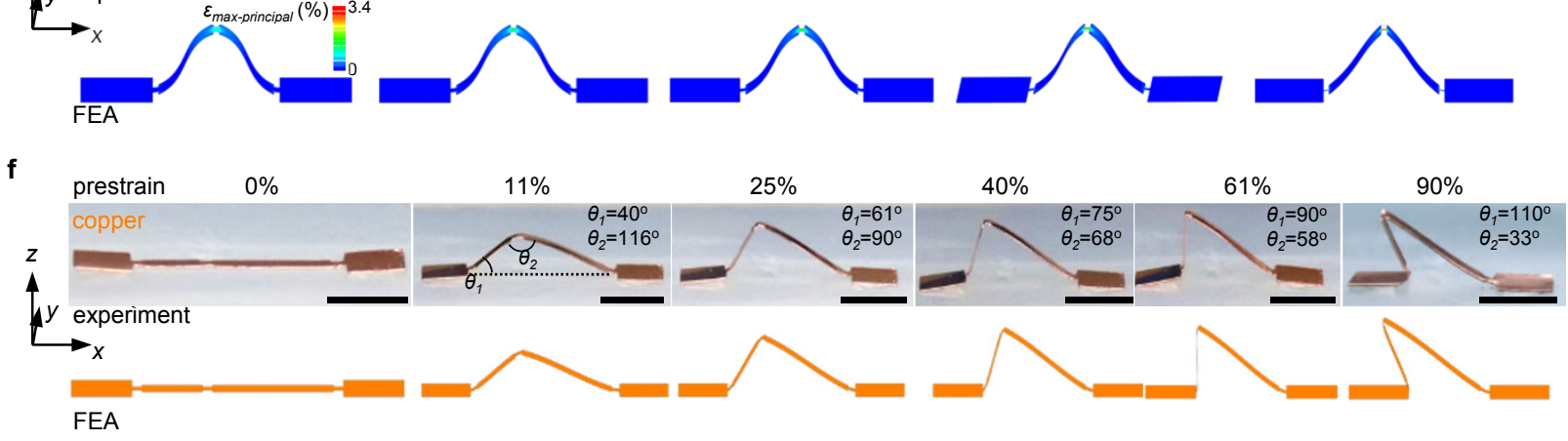

Figure 1 
a

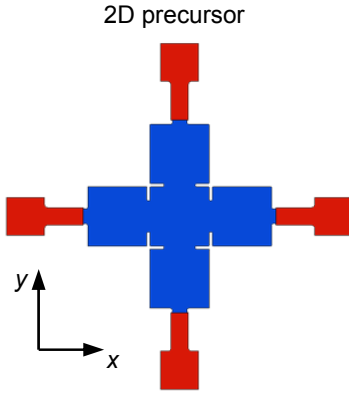

$\stackrel{2}{\longrightarrow}$

b

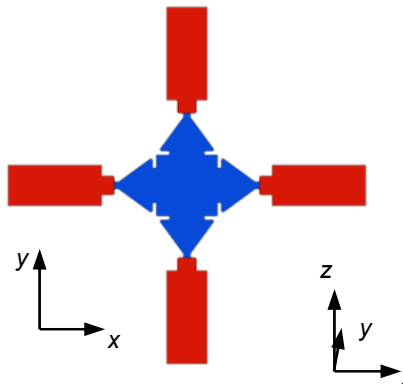

C

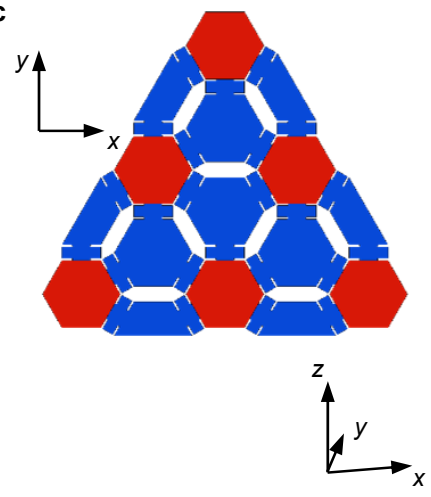

3D structure (experiment)
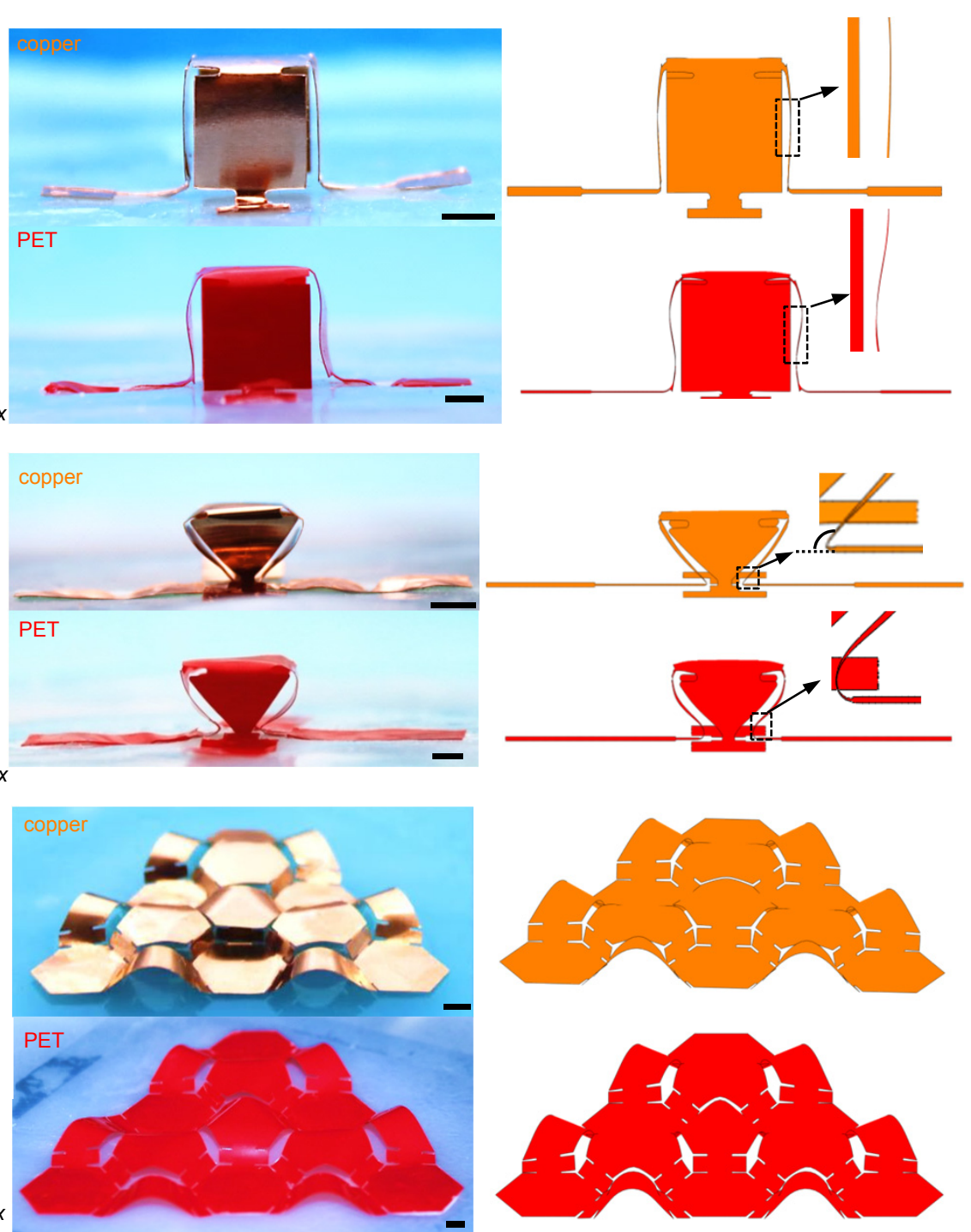

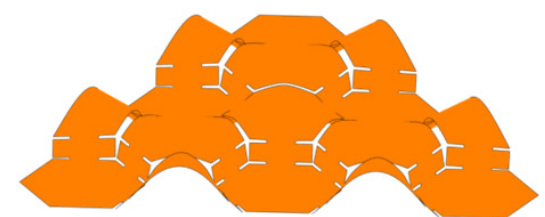

3D structure (FEA)

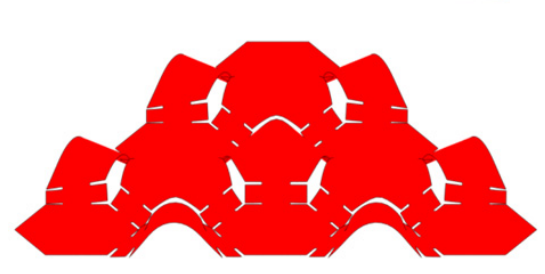

Figure 2 


\begin{tabular}{|l|l|l|l|l|l|}
\hline 2D precursor & $\begin{array}{c}\text { 3D millimeter-scale } \\
\text { structure (experiment) }\end{array}$ & $\begin{array}{c}\text { 3D millimeter-scale } \\
\text { structure (FEA) }\end{array}$ & 2D precursor & $\begin{array}{c}\text { 3D millimeter-scale } \\
\text { structure (experiment) }\end{array}$ & $\begin{array}{c}\text { 3D millimeter-scale } \\
\text { structure (FEA) }\end{array}$ \\
\hline a & & & & &
\end{tabular}

\begin{tabular}{|l|c|c|c|c|c|}
\hline 2D precursor & $\begin{array}{c}\text { 3D mesoscale structure } \\
\text { (experiment) }\end{array}$ & $\begin{array}{c}\text { 3D mesoscale } \\
\text { structure (FEA) }\end{array}$ & 2D precursor & $\begin{array}{c}\text { 3D mesoscale structure } \\
\text { (experiment) }\end{array}$ & $\begin{array}{c}\text { 3D mesoscale } \\
\text { structure (FEA) }\end{array}$ \\
\hline $\mathbf{k}$ & & & & &
\end{tabular}

Figure 3 


\section{Supplementary Information for}

\section{Plasticity-induced origami for assembly of three dimensional metallic structures guided by compressive buckling}

Yan Shi ${ }^{1, \dagger}$, Fan Zhang ${ }^{1, \dagger}$, Kewang $\mathrm{Nan}^{2}$, Xueju Wang ${ }^{3}$, Juntong Wang ${ }^{2}$, Yijie Zhang ${ }^{2}$, Yutong Zhang $^{2}$, Haiwen Luan ${ }^{4}$, Keh-Chih Hwang ${ }^{1}$, Yonggang Huang ${ }^{4}$, John A. Rogers ${ }^{5, *}$, and Yihui Zhang ${ }^{1, *}$

${ }^{1}$ Department of Engineering Mechanics; Center for Mechanics and Materials; Applied Mechanics Laboratory; Tsinghua University, Beijing 100084, P.R. China.

${ }^{2}$ Department of Mechanical Science and Engineering, University of Illinois at Urbana-Champaign, Urbana, Illinois 61801, USA.

${ }^{3}$ Department of Materials Science and Engineering, Northwestern University, Evanston, IL 60208, USA.

${ }^{4}$ Departments of Civil and Environmental Engineering, Mechanical Engineering, and Materials Science and Engineering, Northwestern University, Evanston, IL 60208, USA

${ }^{5}$ Departments of Materials Science and Engineering, Biomedical Engineering, Chemistry, Mechanical Engineering, Electrical Engineering and Computer Science, and Neurological Surgery; Center for Bio-Integrated Electronics; Simpson Querrey Institute for Nano/biotechnology; Northwestern University, Evanston, IL 60208, USA

$\dagger$ These authors contributed equally to this work.

Correspondence and requests for materials should be addressed to John A. Rogers (email: jrogers@illinois.edu) or Yihui Zhang (email: yihuizhang@tsinghua.edu.cn). 


\section{Fabrication of millimeter-scale 3D copper structures}

2D precursors in copper $(30 \mu \mathrm{m})$ and PET $(42 \mu \mathrm{m})$ films were formed by automated mechanical cutting. To avoid undesirable deformation during cuting, the films were adhered to one side of a thermal release tape (releasing temperature, $100{ }^{\circ} \mathrm{C}$ ) with the other side of the tape stuck on an adhesive cutting mat. After heating $\left(100{ }^{\circ} \mathrm{C}\right)$ for about one minute, the patterns were separated from the tape automatically.

A thin silicone substrate (2 $\mathrm{mm}$ in thickness, Dragon Skin) served as the assembly platform. A commercial adhesive (706 RTV silicone rubber, Liyang Kangda Chemical Co., Ltd., Liyang, China), dispensed at desired locations (bonding parts) on the 2D precursors resulted in strong bonding to the silicone substrate, after curing for $\sim 6$ hours at room temperature. Slowly releasing the prestrain in the substrate, with a strain rate $<0.008 \mathrm{~s}^{-1}$, completed the assembly process.

\section{Fabrication of mesoscale 3D copper structures}

Fabrication of mesoscale 3D metallic structures began by preparation of glass substrates for photolithography. This process involved spin-coating 1:4 polydimethylsiloxane (PDMS, Sylgard 184, Dow Corning) at $3000 \mathrm{rpm}$ onto clean glass slides. After curing at $70{ }^{\circ} \mathrm{C}$ for 4 hours, $3 \mu \mathrm{m}$-thick copper foils (MicroThin - $\mathrm{EX}^{\mathrm{TM}}$, Mitsui Mining \& Smelting Co. Ltd.) were laminated onto the PDMS-coated glass. A thin layer of polyimide ( $1 \mu \mathrm{m}, \mathrm{PI}-2545$, MicroSystems) was spin-cast and cured $\left(250{ }^{\circ} \mathrm{C}, \sim 1\right.$ hour) on one side of the copper foils to facilitate flat and bubble-free contacts with the PDMS. Copper was then patterned using standard photolithography followed by wet etching (CE-100 
etchant, Transene, etchant rate $\sim 1 \mu \mathrm{m} / \mathrm{min})$. The samples were then etched using oxygen plasma to remove the exposed polyimide, followed by immersion in diluted 1-Methyl-2-pyrrolidone to fully strip photoresist. Water-soluble, polyvinyl alcohol (PVA) tapes were used to retrieve samples from the glass substrate.

With samples still on the PVA tape, a thin layer of silicon dioxide $(\sim 50 \mathrm{~nm})$ was deposited on the bonding sites by electron-beam evaporation through an aligned shadow mask. After UV-ozone treatment of both the stretched silicone substrate $(\sim 0.5 \mathrm{~mm}$ thick, Dragon Skin ${ }^{\circledR}$, Smooth-On) and the sample surfaces using a UV ozone cleaner (UVOCS® Inc.), the samples were laminated onto the silicone substrate, followed by baking $\left(70^{\circ} \mathrm{C}, 5\right.$ min) in a convection oven. Rinsing with warm water to dissolve the PVA tape, and slowly releasing the pre-strain in the stretched silicone substrate completed the fabrication of mesoscale 3D metallic structures.

\section{Methods: Finite element analyses}

Three-dimensional (3D) finite element analyses (FEA) were employed to simulate the final configurations and strain distributions of the origami structures enabled by controlled buckling. Four-node shell elements were used to model the origami structures. Convergence of mesh sizes was tested to ensure computational accuracy. The critical buckling strains and corresponding buckling modes determined from linear buckling analyses were implemented as initial imperfections in the postbuckling calculations to obtain the deformed configurations and strain distributions at different levels of prestrain. The calculations for the postbuckling process were performed using conventional static analysis 
in the commercial software ABAQUS. An ideally, elastic-plastic constitutive relation was used for the copper film, in which the elastic modulus $(E)$ and Poisson's ratio $(v)$ are $E_{C u}=$ $119 \mathrm{GPa}$ and $v_{C u}=0.34$, with an yielding stress of $357 \mathrm{MPa}$ (corresponding to $0.3 \%$ yielding strain). The material properties for the PET film include $E_{P E T}=2.7 \mathrm{GPa}$ and $v_{P E T}=0.38$. 